e-J. Surf. Sci. Nanotech. Vol. 4 (2006) 69-73

Conference - ISSS-4 -

\title{
Growth mechanism and size effect on fluorescence properties of perylene nanocrystals*
}

\author{
Yutaka Wakayama ${ }^{\dagger}$ and Tadashi Mitsui \\ Nanomaterials Laboratory, National Institute for Materials Science 1-1 Namiki, Tsukuba 305-0044, Japan
}

Tsunenobu Onodera, Hidetoshi Oikawa, and Hachiro Nakanishi Institute of Multidisciplinary Research for Advanced Materials,

Tohoku University, 2-1-1 Katahira, Aoba-ku, Sendai 980-8577, Japan

(Received 12 October 2005; Accepted 26 December 2005; Published 19 January 2006)

\begin{abstract}
The main aims of this study were to explore a new technique for growing molecular nanocrystals and to examine their optical properties. To grow nanocrystals, we applied a vacuum evaporation technique. Small perylene nanocrystals (ca. $200 \mathrm{~nm}$ ) at a high density $\left(7.6 \mu \mathrm{m}^{-2}\right.$ ) were produced by ultra-rapid evaporation on a patternindented Si surface. A perylene-3,4,9,10-tetracarboxylic-3,4,9,10-dianhydride (PTCDA) buffer layer was found to be effective for further downsizing of the nanocrystals and for preserving their fluorescence properties originating from the nanoscale size effect. [DOI: 10.1380/ejssnt.2006.69]
\end{abstract}

Keywords: aromatic molecules; nanoparticles; growth; photoluminescence

\section{INTRODUCTION}

Nanoscale particles, whose dimensions fall between atoms and bulk crystal, have been intensively investigated for their three-dimensional confinement effects. Vacuum techniques have advantageous features for producing nanocrystals, since they allow a wide choice of substrate and nanocrystal materials as well as adjustment of the growth process parameters. The so-called StranskiKrastanov mode is a common means of fabrication of semiconductor quantum dots. The interaction between the substrate and nanocrystals has a crucial effect on both the mechanism of growth of nanocrystals and their optoelectronic properties. Thus, a suitable choice of the parameters allows fine-tuning of both the nanocrystal dimensions and nanoscale size effects.

On the other hand, there is still room to improve the growth techniques of nanoparticles consisting of organic materials. Organic materials demonstrate various optoelectronic functions that depend their dimensions, including single-molecule [1-3], nanocrystal [4] and thin films $[5,6]$. In many cases, the growth mechanism and properties of organic nanocrystals have been investigated only in liquid dispersions [7-9] or as cast films on solid surfaces [4]. However, the growth of organic nanocrystals directly on solid substrates needs to be examined to identify properties related to size effects and to gain an understanding of the chief effects on the growth mechanism of the nanocrystal-substrate interaction. The main purpose of this study centered on the exploration of a new growth technique for molecular nanocrystals and on an examination of the optical properties of these nanocrystals in relation to size effects. We applied a vacuum technique for this purpose because of its advantages in control over the growth of the crystals over a wide variety of experimental parameters, including deposition rate, substrate temperature and surface treatment of the substrates [10].

* This paper was presented at International Symposium on Surface Science and Nanotechnology (ISSS-4), Saitama, Japan, 14-17 November, 2005.

$\dagger$ Corresponding author: WAKAYAMA.Yutaka@nims.go.jp
These experiments were first carried out under extreme conditions, that is, ultra-rapid evaporation under vacuum. This technique provided a nonequilibrium environment for crystal growth. Second, a lithographicallypatterned Si substrate and a molecular buffer layer were employed to fine-tune the nucleation probability and surface diffusion. Finally, the critical effect of the substratenanocrystal interaction on the optical properties was elucidated by measuring the fluorescence spectra of the thus prepared nanocrystals.

\section{EXPERIMENTAL}

Perylene molecules were deposited from an alumina crucible at a background pressure of $1 \times 10^{-4} \mathrm{~Pa}$. A quartz thickness monitor was used to determine the deposition rate and thickness. The substrate was kept at room temperature during the deposition. Equally-spaced dimples were formed on the Si substrates using an electron lithography technique. The patterned Si substrates were then cleaned with alcohol solvents under ultrasonic conditions; a native oxide layer covered their surfaces. Flat Si substrates were also used for comparison. The size, shape and density of the perylene nanocrystals were observed by scanning electron microscopy (SEM; JEOL Ltd., JSM-6500). The crystallinity and orientation of the thus prepared perylene nanocrystals were examined by X-ray diffractometry (XRD; Brucker AXS, D8 Discover Super Speed). For fluorescence measurements, a Ti: sapphire laser was used as a light source. The samples were irradiated at an excitation wavelength of $385 \mathrm{~nm}$. The fluorescence spectra were obtained at room temperature.

\section{RESULTS AND DISCUSSION}

\section{A. Growth of perylene nanocrystals}

Figure 1 shows the molecular structure of perylene and molecular packing of $\alpha$-perylene unit cells. The SEM images in Figs. 2(a)-(f) show the dependency of shape, size and density of the perylene nanocrystals on the deposition 
(a)

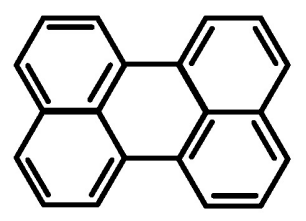

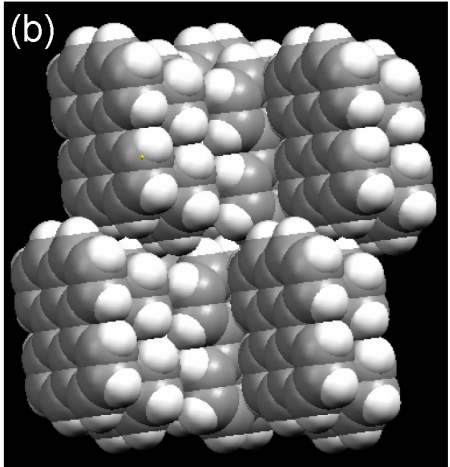

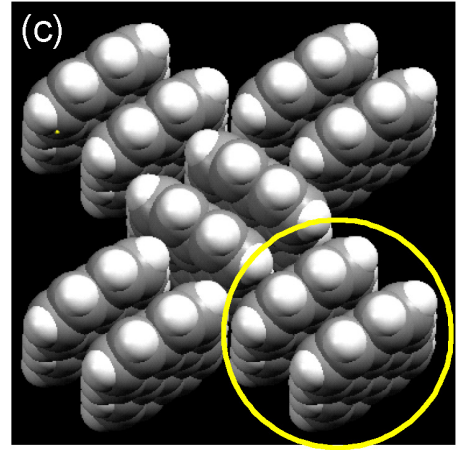

FIG. 1: (a) Molecular structure of perylene. Molecular packing of the $\alpha$-perylene unit cell viewed from (b) the [010] direction and (c) the [001] direction. Each lattice point is occupied by two molecules, encircled in (c).
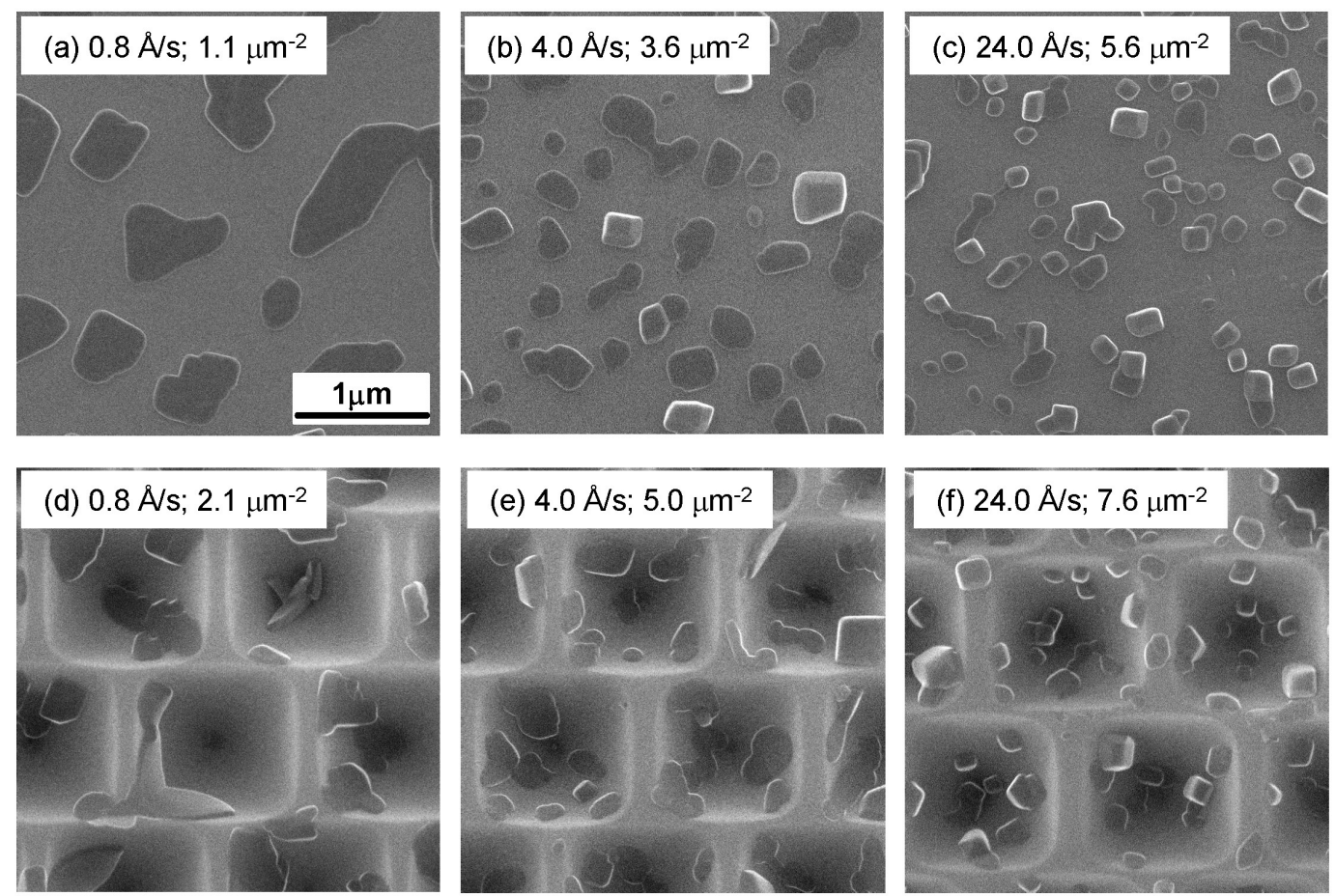

FIG. 2: SEM images of perylene deposited at different rates ((a) and (d), $0.8 \AA /$ sec; (b) and (e), $4.0 \AA$ Asec; and (c) and (f), $24.0 \AA / \mathrm{sec}$ ) on flat substrates ((a), (b) and (c)) and on patterned substrates ((d), (e) and (f)). The number densities of each specimen are indicated in each image.

rate and on the substrate figuration. Nominal thickness was kept at $12.0 \mathrm{~nm}$ for all samples.

Figures 2(a)-(c) show perylene crystals on the flat $\mathrm{Si}$ substrates formed at different deposition rates, which were $0.8 \AA / \mathrm{sec}, 4.0 \AA / \mathrm{sec}$ and $24.0 \AA / \mathrm{sec}$, respectively. The relatively slow deposition rate $(0.8 \AA / \mathrm{sec})$ produced disk-like grains as shown in Fig. 2(a). The size of the grains ranges from $0.5 \mu \mathrm{m}$ to $1.0 \mu \mathrm{m}$. The shape of the grains changed from disk-like to cubic with increasing deposition rate (see Figs. 2(a)-(c)). The number density of the grains simultaneously increases from 1.1 to $5.6 \mu \mathrm{m}^{-2}$. These results indicate that the increase in deposition rate gives rise to an increase in the nucleation probability, restricting grain growth in the lateral direction.

Figures 2(d)-(f) show SEM images of the perylene nanocrystals deposited at the three rates on the surface- indented Si substrates. A comparison of Figs. 2(a) and 2 (d) reveals that on the patterned substrate, grain growth in the lateral directions was suppressed. The size of the grains varies widely, although most are less than $0.5 \mu \mathrm{m}$ in size. Regardless of the deposition rate, the patterned surface induced a decrease in the size of the nanocrystals and an increase in density as compared with the flat substrates (compare Figs. 2(b) and 2(d), Figs. 2(c) and $2(\mathrm{f}))$.

The extremely high deposition rate of $24.0 \AA /$ sec produces an excessive concentration of molecules diffusing on the surface, which provides the supersaturated environment for crystal growth. This condition causes frequent collisions among molecules and a consequent increase in the probability of nucleation, resulting in nanocrystals with small sizes and high number density. Meanwhile, 

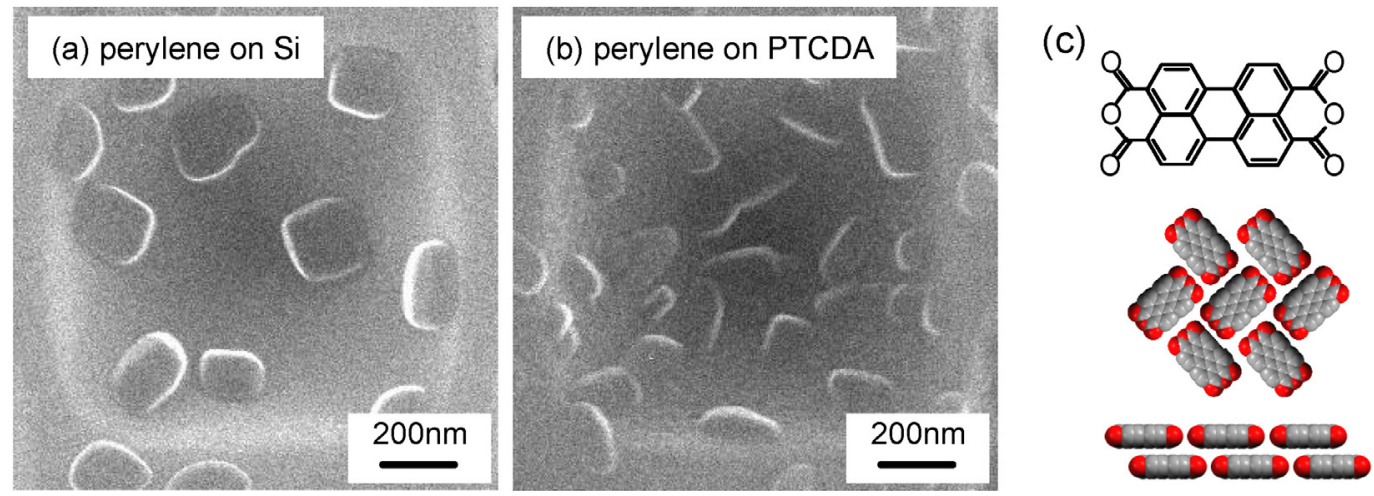

FIG. 3: SEM images of perylene nanocrystals (a) grown on Si and (b) on a PTCDA buffer layer. The deposition rate of perylene was $24.0 \AA / \mathrm{sec}$ for both specimens. (c) The molecular structure of PTCDA, top and side view of the molecular arrangement of the PTCDA (102) plane are illustrated. Red circles denote oxygen atoms.

(a) perylene on $\mathrm{Si}$

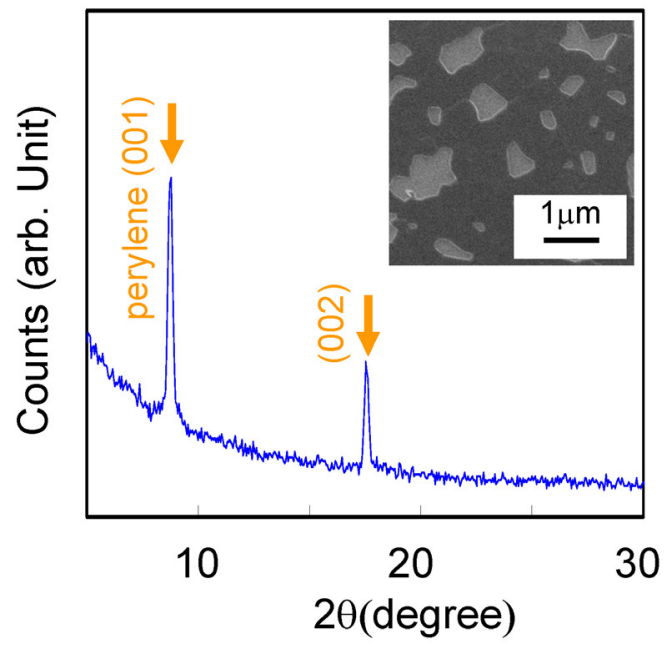

(c)

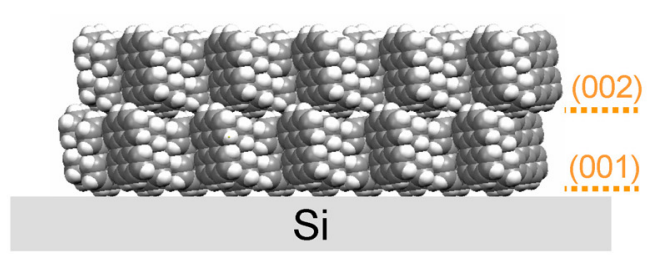

(b) perylene on PTCDA

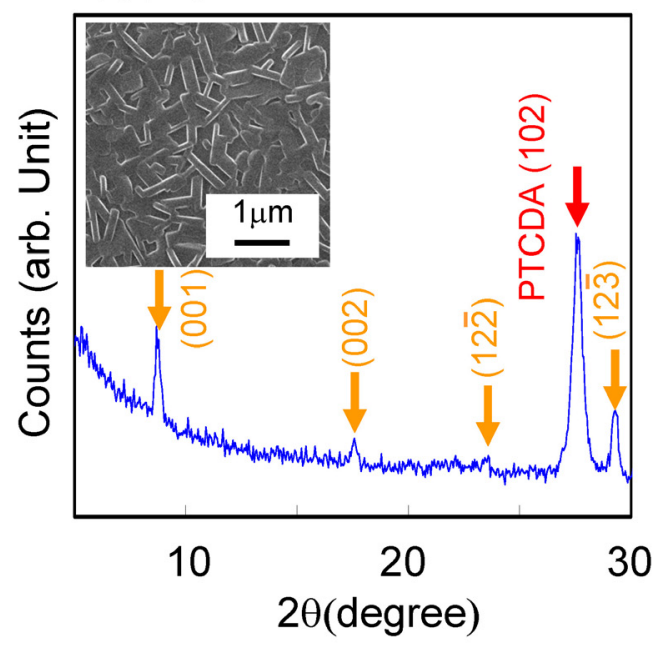

(d)

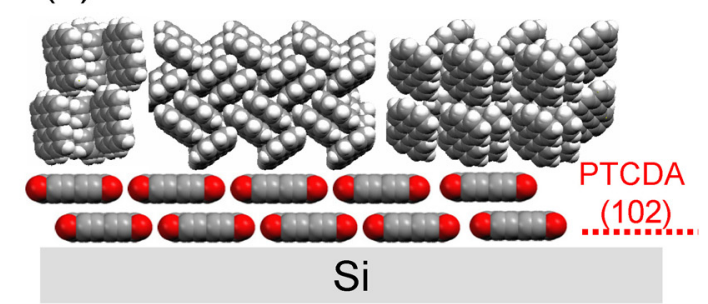

FIG. 4: XRD patterns and SEM images of perylene (a) on Si and (b) on PTCDA. Perylene with a thickness of $60 \mathrm{~nm}$ was deposited on the flat Si substrates at a deposition rate of $24.0 \AA /$ sec. The illustrations describe (c) $\alpha$-perylene highly oriented to the (001) plane and (d) randomly oriented $\alpha$-perylene on the (102) plane of PTCDA.

high diffusivity on the surface simultaneously leads to a larger grain size. The pattern-indented surface was designed to suppress surface diffusion and thus prevent lateral crystal growth. As a result, a combination of ultrarapid deposition and a patterned surface was found to be advantageous for producing perylene nanocrystals with a small size (ca. $200 \mathrm{~nm})$ and a high density $\left(7.6 \mu \mathrm{m}^{-2}\right)$, as shown in Fig. 2(f).

The surface treatment of substrates has been reported to be effective in both modifying the growth mechanism and promoting optoelectronic functionalities
$[11,12]$. In this study, the effect of an underlying buffer layer was examined to explore the controllability of the nanocrystal growth and the effect on the fluorescence properties. For this purpose, we utilized a perylene derivative, perylene-3,4,9,10-tetracarboxylic3,4,9,10-dianhydride (PTCDA), because this molecule forms two-dimensional continuous films, covering up the surface $[12,13]$. The film then grows with a preferential orientation in the (102) plane, where the molecular plane is parallel to the surface, which enables effective surface modification. 
(a)

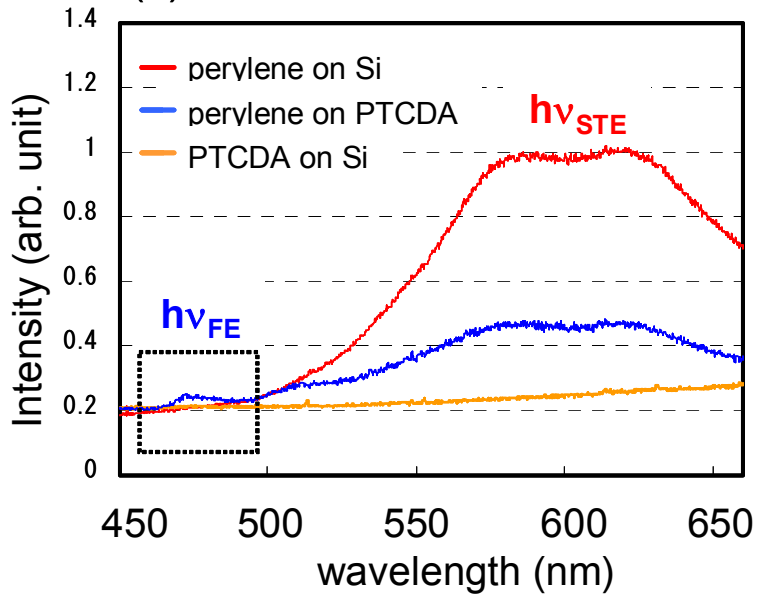

(b)

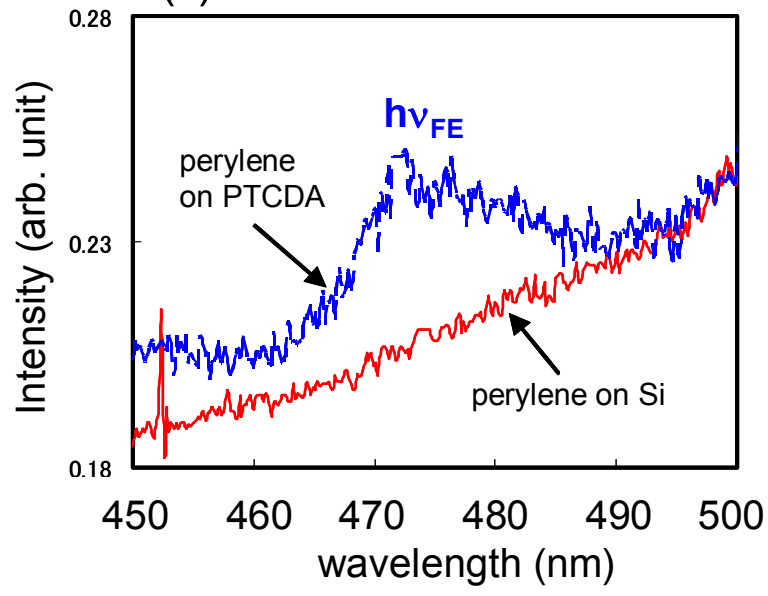

FIG. 5: (a) Fluorescence spectra of the perylene nanocrystals on Si and on PTCDA. The broad bands ranging from 530 nm-660 $\mathrm{nm}$ and the weak emission at $460 \mathrm{~nm}-490 \mathrm{~nm}$ come from self-trapped $\left(h \nu_{S T E}\right)$ and free excitons $\left(h \nu_{F E}\right)$, respectively. No light emission was detected from the PTCDA buffer layer, as shown by the yellow line. (b) Enlarged spectra ranging from 450 nm-500 nm clearly show distinct differences between the two spectra. Only perylene on PTCDA shows $h \nu_{F} E$.

Figures 3(a) and 3(b) are SEM images of perylene nanocrystals deposited directly onto a Si surface and onto a PTCDA buffer layer, respectively. In Fig. 3(b), a PTCDA underlying film with a thickness of $20 \mathrm{~nm}$ was deposited prior to perylene deposition. The molecular structure of PTCDA and the top and side views of molecular arrangement in the (102) plane are illustrated in Fig $3(\mathrm{c})$. The nominal thickness $(12 \mathrm{~nm})$ and deposition rate $(24.0 \AA / \mathrm{sec})$ of perylene are the same as those in Fig. 2. These SEM images revealed that the PTCDA buffer layer had several distinct influences on the perylene nanocrystal growth. First, a clear increase in the number density of the perylene nanocrystals was found. The density of the perylene nanocrystals on the PTCDA buffer layer was determined to be $13.6 \mu \mathrm{m}^{-2}$. Second, a decrease in size was observed, ranging from $50 \mathrm{~nm}$ to $200 \mathrm{~nm}$, with an average of roughly $100 \mathrm{~nm}$. Additionally, the nanocrystals in this image are irregular in shape.

The following experiments were carried out to study the growth mechanism of the perylene nanocrystals, and especially to clarify the influence of the PTCDA buffer layer. Flat Si substrates were used and 60 nm-thick perylene layers were deposited, both directly on the Si substrate and on the PTCDA buffer layers. The deposition rate was $24.0 \AA / \mathrm{sec}$, the same as in the previous experiment. Clear differences in the morphology and crystallinity of the perylene layers were found in XRD patterns and SEM images, as shown in Figs. 4(a) and (b). The SEM image in the inset of Fig. 4(a) shows that perylene on Si formed a flat, two-dimensional (2D) structure. In the XRD pattern of this specimen, only two peaks were observed (at $8.80^{\circ}$ and $17.55^{\circ}$ ), which revealed that the perylene layer was highly oriented in the [001] direction of the unit cell. This result indicates that the molecules have a standing-up orientation and the molecular plane is almost perpendicular to the substrate surface [14]. Figure 4(c) is a schematic illustration of the orientation of the perylene molecules on the Si substrate.

Based on this, the arrangement of the perylene nanocrystals can be regarded as due to the early stages of $2 \mathrm{D}$ growth being oriented with the [001] plane. The cubic shape of the nanocrystals is a reflection of the shape of the unit cell of $\alpha$-perylene crystal (P $2_{1} / \mathrm{c}$ ). On the other hand, perylene on the PTCDA grew with a characteristic rod-like structure [15], as can be seen in the inset of Fig. 4(b), implying that perylene on PTCDA has less wettability than on Si. The several peaks observed in the XRD pattern (Fig. 4(b)) reveal that the perylene molecules were randomly orientated. The strong diffraction peak at $27.6^{\circ}(\mathrm{d}=3.22 \AA)$ originates from the PTCDA (102) plane. These results indicate that less wettability of the perylene on PTCDA caused the small size, irregular shape and random orientation of the perylene nanocrystals. Figures $4(\mathrm{~d})$ is a diagram of the random orientation of the perylene nanocrystals on the well-ordered PTCDA (102) plane.

\section{B. Fluorescence properties of perylene nanocrystals}

Perylene nanocrystals have been reported to show sizedependent optical properties [4]. In this study, we examined its fluorescence properties as a function of the perylene-substrate interaction. In Fig. 5, the fluorescence spectra of perylene nanocrystals grown on $\mathrm{Si}$, those on a PTCDA buffer layer, and PTCDA alone on Si are shown in red, blue, and yellow, respectively. We expected that light emissions from both self-trapped excitons (STEs) and free excitons (FEs) would be present because the size of these crystals is small enough to show a lattice softening that causes an energy relaxation through FE $[4,8]$ in addition to STEs. On the contrary, perylene nanocrystals grown directly on the Si substrate gave only STE light emission (red spectrum), the wavelength of which ranged from $530 \mathrm{~nm}$ to $660 \mathrm{~nm}$. Meanwhile, those on the PTCDA buffer layer exhibited fluorescence from both self-trapped and free excitons (blue spectrum). When the fluorescence spectra were enlarged, FE light emission 
was clearly present, with the wavelength peak at $472 \mathrm{~nm}$. These results suggest that the softening of the perylene nanocrystals was preserved by the PTCDA buffer layer.

The fluorescence properties of a crystal are closely related to its crystal lattice structure. As illustrated in Fig. 1(c), dual molecules with a face-to-face configuration occupy one lattice point, making it easy to form an excimer in the excited state. A self-trapped exciton consists of these dual molecules, similar to an excimer $[4,8,16]$. Light emission from STEs $\left(h \nu_{S T E}\right)$ is dominant in the nanocrystals on $\mathrm{Si}$, since the lattice distortion caused by interacting with the Si substrate makes it possible to reach the lattice relaxation energy. On the other hand, this energetic gain is still small if lattice softening is preserved due to the size effect associated with the nanocrystals. As a result, light emission from both FEs $\left(h \nu_{F E}\right)$ and STEs $\left(h \nu_{S T E}\right)$ is permitted due to the small energy barrier between STEs and FEs. A detailed discussion of this phenomenon is described elsewhere using coordination energy diagrams [4].

In this study, the size of the nanocrystals is not crucial in determining the fluorescence property, but the interaction between the nanocrystal and the substrate is the essential factor. As discussed above, the perylene nanocrystals are adsorbed onto $\mathrm{Si}$ with a high wettability. The strong adsorption induced lattice distortion that decreased the coordination energy. This reduction in the coordination energy restrained the energy relaxation through FEs. Consequently, only $h \nu_{S T E}$ were observed in the fluorescence spectrum, in a similar manner to that of bulk crystals, in spite of the crystals being small enough to allow lattice softening. In contrast, the PTCDA buffer layer induced $h \nu_{F E}$ by preventing the di- rect interaction of the crystals with the Si substrate. The PTCDA buffer layer is thought to reduce the distortion of the nanocrystal lattice. It can therefore be concluded that the PTCDA buffer layer has two apparent effects: the downsizing of the nanocrystals plus a fluorescence signal originating from the size effect.

\section{CONCLUSIONS}

The growth mechanisms of perylene nanocrystals and their optical properties were investigated. The combination of ultra-rapid evaporation, a pattern-indented surface, and a PTCDA buffer layer achieved growth of small nanocrystals at a high density of $13.6 \mu \mathrm{m}^{-2}$. The nucleation probability was increased by ultra-rapid evaporation. The surface diffusion of the deposited molecules is restricted by using a patterned substrate. The PTCDA buffer layer is responsible for the reduction of the interaction of perylene with the substrate. As a result, a further decrease in size and increase in density, as well as the characteristic fluorescence of nano-sized crystals, were achieved.

\section{Acknowledgments}

This study was carried out as part of the "R\&D Promotion Scheme Funding International Joint Research" promoted by the National Institute of Information and Communications Technology.
[1] S. Kubatkin, A. Danilov, M. Hjort, J. Cornil, J. L. Bedas, N. S. Hansen, P. Hedegard, T. Bjernholm, Nature 425, 698 (2003).

[2] T. Kubota, S. Yokoyama, T. Nakahama, S. Mashiko, Y. Noguchi, Y. Majima, M. Iwamoto, Thin Solid Films 393, 379 (2001).

[3] Y. Wakayama, T. Kubota, H. Suzuki, T. Kamikado, S. Mashiko, J. Appl. Phys. 94, 4711 (2003).

[4] H. Oikawa, T. Mitsui, T. Onodera, H. Kasai, H. Nakanishi, T. Sekiguchi, Jpn. J. Appl. Phys. 42, L111 (2003).

[5] G. Witte, C. Wöll, J. Mater. Res. 19, 1889 (2004).

[6] C. D. Dimitrakopoulos, D. J. Mascaro, IBM J. Res. \& Dev. 45, 11 (2001).

[7] Y. Kaneko, T. Onodera, H. Kasai, S. Okada, H. Oikawa, H. Nakanishi, T. Fukuda, H. Matsuda,. J. Mater. Chem. 15, 253 (2005).

[8] T. Seko, K. Ogura, Y. Kawakami, H. Sugino, H. Toyotama, J. Tanaka, Chem. Phys. Lett. 291, 438 (1998).

[9] H. Kasai, H. Kamatani, S. Okada, H. Oikawa, H. Mat- suda, H. Nakanishi, Jpn. J. Appl. Phys. 35, L221 (1996).

[10] S. Verlaak, S. Steudel, P. Heremans, Phys. Rev. B 68, 195409 (2003).

[11] D. S. Park, S. J. Kang, H. J. Kim, M. H. Jang, M. Noh, K.-H. Yoo, C. N. Whang, M. H. Lee, J. Vca. Sci. Technol. B 23, 926 (2005).

[12] S. Heutz, R. Cloots, T. S. Jones, Appl. Phys. Lett. 77, 3938 (2000).

[13] S. Mannsfeld, T. T. Schmitz-Hübsch, F. Sellam, T. Fritz, K. Leo, Organic Electronics 2, 121 (2001).

[14] X. Liu, V. Kaiser, M. Wuttig, T. Michely, J. Crys. Growth 269, 542 (2004).

[15] T. Onodera, T. Oshikiri, H. Katagi, H. Kasai, S. Okada, H. Oikawa, M. Terauchi, M. Tanaka, H. Nakanishi, J. Crys. Growth 229, 586 (2001).

[16] R. Mizoguchi, S. S. Kano, A. Wada, Chem. Phys. Lett. 379, 319 (2003). 